\title{
Netrin/DCC Signaling Controls Contralateral Dendrites of Octavolateralis Efferent Neurons
}

\author{
Arminda Suli, ${ }^{1}$ Nathan Mortimer, ${ }^{3}$ Iain Shepherd, ${ }^{3}$ and Chi-Bin Chien ${ }^{1,2}$ \\ ${ }^{1}$ Department of Neurobiology and Anatomy and ${ }^{2}$ Brain Institute, University of Utah, Salt Lake City, Utah 84132, and ${ }^{3}$ Department of Biology, Emory \\ University, Atlanta, Georgia 30322
}

\begin{abstract}
The guidance molecule Netrin and its receptor DCC (deleted in colorectal cancer) attract commissural axons toward the midline en route to their final destination. To test whether these molecules can also guide dendrites, we studied the contralateral dendrites of zebrafish octavolateralis efferent (OLe) neurons, which are unusual in that they navigate toward and cross the midline. We found that, at the time of dendrite outgrowth, OLe neurons express $d c c$, and the hindbrain midline expresses netrin1. Knocking down $d c c$ or netrin 1 function by injecting antisense morpholino oligonucleotides prevented OLe contralateral dendrites from crossing the midline, showing that $d c c$ and netrin 1 are necessary for dendrite guidance or formation. Furthermore, by transplanting cells from $d c c$ morphants into wild-type embryos and vice versa, we demonstrated that $d c c$ acts cell autonomously in OLe dendrites. This work is the first evidence that Netrin/DCC signaling acts in dendrites in a vertebrate system.
\end{abstract}

Key words: Netrin; DCC; dendritic guidance; zebrafish; hindbrain; octavolateralis

\section{Introduction}

As axons pathfind, several families of guidance molecules guide them toward or away from certain regions (Dickson, 2002). Because dendrites navigate through similar environments, it is plausible that the same guidance molecules could guide them. However, dendrites are difficult to observe because of their short length and proximity to the soma, and so only a few reports have identified dendritic guidance molecules (Polleux et al., 2000; Godenschwege et al., 2002; Furrer et al., 2003; Kim and Chiba, 2004). Here we use the octavolateralis efferent (OLe) neurons in the zebrafish hindbrain, whose midline-crossing dendrites are particularly easy to visualize, to test the role of Netrin/DCC (deleted in colorectal cancer) signaling in guiding vertebrate dendrites.

DCC is a transmembrane protein of the Ig superfamily (Fearon et al., 1990; Hedrick et al., 1994) that acts as a receptor for the guidance cue Netrin to mediate axon attraction (Chan et al., 1996; Keino-Masu et al., 1996; Dickson, 2002). Although Drosophila Frazzled/DCC guides dendrites of aCC motor neurons (Furrer et al., 2003), it has not been tested whether DCC plays a similar role in guiding vertebrate dendrites.

While studying required functions of zebrafish $d c c$ (Hjorth et

Received July 5, 2006; revised Nov. 15, 2006; accepted Nov. 16, 2006.

This work was supported by National Institutes of Health Grant R01 EY12873 (C.-B.C.) and March of Dimes Basil O'Connor Award 5-FY02-270 (I.S.). We thank H. Okamoto and H. Baier for providing the is/1:gfp and brn3c:gap43-gfp fish lines; M. Bak-Maier and S. Fraser for discussing unpublished work, reading the manuscript, and pointing out the misspliced RNA generated by DCC-M03; Lara Hutson for designing DCC-M01; and K. Kwan, A. Pittman, K. Rasband, and J. Rosenthal for help with quantitation. Thanks to A. Alvarado-Sanchez, R. Dorsky, D. Grunwald, T. Piotrowski, and all members of the Chien laboratory for discussions and support and to R. Dorsky and J. Bonkowsky for comments on this manuscript.

Correspondence should be addressed to Chi-Bin Chien, Department of Neurobiology and Anatomy, 401 MREB University of Utah Medical Center, 20 North 1900 East, Salt Lake City, UT 84132-3401. E-mail: chi-bin.chien@neuro.utah.edu.

DOI:10.1523/JNEUROSCI.2858-06.2006

Copyright $\odot 2006$ Society for Neuroscience $\quad 0270-6474 / 06 / 2613328-10 \$ 15.00 / 0$ al., 2001; Fricke and Chien, 2005), we analyzed OLe dendrites, which are unusual in that they project contralaterally, across the hindbrain midline, a domain that expresses netrin1 (Lauderdale et al., 1997; Strahle et al., 1997). In aquatic vertebrates, the OLe system provides efferent innervation to hair cells of the lateral line and ear, whereas in terrestrial vertebrates only the ear is innervated. OLe neurons are considered to be a subset of cranial motor neurons that innervate neuroepithelium rather than muscle (Roberts and Meredith, 1989; Chandrasekhar, 2004). Backlabeling studies show that zebrafish OLe neurons in ventral hindbrain are organized in two clusters: the rostral efferent nuclei (REN) (also known as ROLE) and the caudal efferent nuclei (CEN) (also known as RELL) (Zottoli and Van Horne, 1983; Metcalfe et al., 1985; Bricaud et al., 2001). In some teleosts, including zebrafish, these neurons use an unusual anatomical strategy to integrate bilateral neural input: their cell bodies lie ipsilateral to their targets, whereas their dendrites extend both ipsilaterally and contralaterally (Metcalfe et al., 1985; Roberts and Meredith, 1989; Bricaud et al., 2001). We asked whether Netrin1 from the midline, acting through DCC, might guide the contralateral OLe dendrites.

We visualized OLe neurons using $\operatorname{Tg}(\text { isll:gfp })^{\mathrm{rw} 0}$ transgenic zebrafish, which express green fluorescent protein (GFP) in cranial motor neurons (Chandrasekhar et al., 1997; Higashijima et al., 2000). We first confirmed that GFP-positive hindbrain commissures are indeed OLe dendrites. We then showed that $d c c$ is expressed in REN and CEN as their contralateral dendrites grow toward the midline, in which netrin $1 a$ and netrin $1 b$ are expressed. Knocking down either $d c c$ or netrin 1 function prevented midline crossing by OLe contralateral dendrites, and cell transplant experiments showed that DCC acts cell autonomously in OLe neurons. Together, these data show that the axon guidance molecules Netrin1 and DCC act in dendrites in a vertebrate system. 


\section{Materials and Methods}

Fish stocks and embryo raising. Adult fish were bred according to standard conditions. Tup Longfin was used as the wild-type (WT) strain. $\operatorname{Tg}$ (isl1: $g f p)^{\mathrm{rw0}}$ and $\operatorname{Tg}(b r n 3 c \text { : gap43-gfp })^{\mathrm{s} 356 \mathrm{t}}$ transgenic lines were kind gifts of Hitoshi Okamoto (RIKEN Brain Institute, Wako, Japan) and Herwig Baier (University of California, San Francisco, CA), respectively. Embryos were raised at $28.5^{\circ} \mathrm{C}$ in $\mathrm{E} 3$ embryo medium with $0.003 \%$ phenylthiourea to inhibit pigment formation and staged according to Kimmel et al. (1995). For both in situ and antibody (Ab) staining, embryos were fixed in $4 \%$ paraformaldehyde (PFA) (in PBS) for $1-2 \mathrm{~h}$ at room temperature $(\mathrm{RT})$ or overnight $(\mathrm{O} / \mathrm{N})$ at $4^{\circ} \mathrm{C}$, washed briefly in $\mathrm{PBS}$, dehydrated, and stored in $100 \% \mathrm{MeOH}$ at $-20^{\circ} \mathrm{C}$ until use.

OLe back-labeling from the lateral line. Embryos from a cross of an isl1:gfp transgenic (Higashijima et al., 2000) to a brn3c:gap43-gfp transgenic (Xiao et al., 2005) were anesthetized at $44 \mathrm{~h}$ postfertilization (hpf) in tricaine $(0.16 \%$ final concentration) and embedded laterally in $1.5 \%$ low-melting agarose in E2/GN media. Agarose was removed around the L1 neuromast (labeled by brn3c:gap43-gfp), and a glass micropipette was used to scratch the skin and pressure inject 5\% Alexa-568 dextran (Invitrogen, Carlsbad, CA) using a Picospritzer II (General Valve, Fairfield, $\mathrm{NJ})$. Embryos were removed from agarose and raised at $28.5^{\circ} \mathrm{C}$ until 60 hpf, when they were reembedded and imaged live.

In situ hybridization and anti-GFP staining. Standard in situ hybridization procedures were modified for double staining with anti-GFP. Antisense RNA probe was prepared from clone DCC665, comprising approximately half of the DCC coding sequence, using T7 polymerase and the DIG RNA labeling kit (Roche, Indianapolis, IN). Embryos were rehydrated to PBS, washed briefly with PBST (0.1\% Tween 20 in PBS), digested with $0.1 \%$ collagenase (Sigma, St. Louis, MO) for $15 \mathrm{~min}$, washed with PBST, postfixed for $10 \mathrm{~min}$ in $4 \%$ PFA, treated with $0.25 \%$ acetic anhydride in $0.1 \mathrm{~m}$ triethanolamine, $\mathrm{pH} 7.0$, for $1 \mathrm{~h}$ at $\mathrm{RT}$, hybridized with the RNA probe $\mathrm{O} / \mathrm{N}$ at $65^{\circ} \mathrm{C}$, washed with SSCT (SSC in $0.1 \%$ Tween 20 ) at $65^{\circ} \mathrm{C}$, treated with $10 \mu \mathrm{g} / \mathrm{ml}$ RNaseA at $65^{\circ} \mathrm{C}$ for $30 \mathrm{~min}$ to digest unbound probe, and incubated for at least $2 \mathrm{~h}$ with $2 \%$ blocking reagent (Roche). Embryos were then incubated $\mathrm{O} / \mathrm{N}$ with alkaline phosphatase (AP)-conjugated anti-DIG Fab fragment (1:5000; Roche) and rabbit anti-GFP polyclonal antibody (pAb) (1:400; Invitrogen), washed with PBST, and developed by incubating in AP substrate (Roche) or nitroblue-tetrazolium-chloride/5-bromo-4-chlor-indolyl-phosphate (Roche) in 10\% polyvinyl alcohol. Anti-GFP staining was completed by washing with PBST, incubating $\mathrm{O} / \mathrm{N}$ with HRP-labeled anti-rabbit secondary (1:100; Invitrogen), washing in PBST, incubating briefly with $0.5 \%$ DMSO in PBS, and developing in Alexa-488 tyramide ( $1: 100$; Invitrogen) in $0.0015 \% \mathrm{H}_{2} \mathrm{O}_{2}$ in PBS.

Sectioning. Stained embryos were embedded in $1.5 \%$ agar $/ 5 \%$ sucrose in PBS. The trimmed agar block was sunk in $30 \%$ sucrose $\mathrm{O} / \mathrm{N}$ at $4^{\circ} \mathrm{C}$, then embedded in OCT, and sectioned at $14 \mu \mathrm{m}$ on a Leica (Nussloch, Germany) CM 3050S cryostat. Sections were air dried, coverslipped with Fluoromount-G (Southern Biotechnology, Birmingham, AL), and photographed using a Magnafire SP camera on an Olympus Optical (Melville, NY) BX-50WI microscope.

Anti-GFP staining. Stored embryos were rehydrated to PBS, incubated in $1 \% \mathrm{H}_{2} \mathrm{O}_{2}$ for $1 \mathrm{~h}$ to block endogenous peroxidase, permeabilized with $0.1 \%$ collagenase for $15 \mathrm{~min}$, washed in PBST, postfixed in 4\% PFA for 10 min, washed in PBST, blocked with NCST (10\% heat-inactivated newborn calf serum, $0.1 \%$ Tween 20 , and $1 \%$ DMSO, in PBS) for at least $1 \mathrm{~h}$, and incubated with rabbit anti-GFP pAb (1:400) in NCST, O/N at $4^{\circ} \mathrm{C}$. Embryos were then extensively washed with PBST, incubated for $5 \mathrm{~h}$ at room temperature with HRP anti-rabbit secondary (1:100) in NCST, washed in PBST, treated with 0.5\% DMSO in PBS, and developed in Alexa-488 tyramide (1:100; Invitrogen) in $0.0015 \% \mathrm{H}_{2} \mathrm{O}_{2}$ in PBS.

Microtubule-associated protein staining. Embryos were fixed in 2\% trichloroacetic acid, washed with PBST (0.5\% Triton X-100 in PBS), blocked with NCST, and incubated with anti-microtubule-associated protein 2 (MAP2) (2a and 2b) Ab (1:100; BD PharMingen, San Jose, CA) and anti-GFP pAb (1:400) in NCST O/N at $4^{\circ} \mathrm{C}$, washed with PBST, incubated for $4 \mathrm{~h}$ at RT with anti-rabbit Alexa-488 secondary (1:100; Invitrogen) and anti-mouse HRP (1:100) in NCST, washed in PBST, treated with $0.5 \%$ DMSO in PBS, and developed in Alexa-568 tyramide ( $1: 100$; Invitrogen) in $0.0015 \% \mathrm{H}_{2} \mathrm{O}_{2}$ in PBS.

Morpholino oligonucleotide sequences and reverse transcription-PCR. All morpholino oligonucleotides (MOs) were synthesized by Gene Tools (Philomath, OR). Control MO was a standard control from Gene Tools: 5'-CCTCTTACCTCAGTTACAATTTATA-3'. MOs were designed using published $d c c$ and netrin coding sequences, $d c c 5^{\prime}$ untranslated region (UTR) sequence (C. Fricke and C. B. Chien, unpublished observation), and genomic sequence (Sanger Centre, Hinxton, Cambridge, UK): DCC-MO1, GAATATCTCCAGTGACGCAGCCCAT $(+1$ to $+25 \mathrm{bp}$, reverse-strand start codon underlined); DCC-MO2, GCGAAATCCGCTGCTAATCAATCAA ( -42 to $-18 \mathrm{bp})$; DCC-MO3, GAGCAGCACTGACCGTGTGTGTAGA (last $12 \mathrm{bp}$ of exon 1 and first $13 \mathrm{bp}$ of intron 1); netrin1a splice blocking MO, ATGATGGACTTACCGACACATTCGT (last 11 bp of exon 1 and first 14 bp of intron1); and netrin $1 b$ translation blocking MO, CGCACGTTACCAAAATCCTTATCAT $(+1$ to $+25 \mathrm{bp}$ ).

Efficacy of splice-blocking MOs was assayed by reverse transcriptionPCR from total RNA of 10 pooled injected embryos. Unspliced $d c c$ premRNA was amplified using primers P1 (TGCGTCACTGGAGATATTCG) and P2 (GTCATGCGCTGCTTCAATTA). Correctly spliced $d c c$ mRNA was amplified using P1 and P3 (GAGGCCAGACACTGGTAGGA). As a loading control, $\beta$-actin mRNA was amplified using $\beta$-actin FP (CCCAAGGCCAACAGGGAAAA) and $\beta$-actin RP (GGTGCCCATCTCCTGCTCAA). PCR conditions were as follows: 2 min $94^{\circ} \mathrm{C}$; followed by 35 cycles $(d c c)$ or 22 cycles $\left(\beta\right.$-actin) of $94^{\circ} \mathrm{C}, 30 \mathrm{~s} / 59^{\circ} \mathrm{C}$, $30 \mathrm{~s} / 72^{\circ} \mathrm{C}, 60 \mathrm{~s}$; followed by $5 \mathrm{~min} 72^{\circ} \mathrm{C}$. netrin $1 \mathrm{a}$ pre-mRNA was amplified with primers F1 (CTTTCGGAGACGAAAACGAG) and R1 (GTAGGCGCTTTCCAGAGATG); netrin 1a mRNA was amplified with primers F1 and R2 (CTTTGCAGTAGTGGCAGTGG). PCR conditions were as follows: 2 min $94^{\circ} \mathrm{C}$; followed by 35 cycles (netrin1a) or 22 cycles $(\beta$ actin) of $94^{\circ} \mathrm{C}, 30 \mathrm{~s} / 59^{\circ} \mathrm{C}, 30 \mathrm{~s} / 72^{\circ} \mathrm{C}, 60 \mathrm{~s}$; followed by 5 min $72^{\circ} \mathrm{C}$.

MO injections. Lyophilized MOs were diluted in Danieau's solution and stocks were aliquoted; concentrations were checked by spectrophotometry (A265 in $0.1 \mathrm{~N} \mathrm{HCl}$, according to the protocol of Gene Tools). Working solutions were diluted in water and phenol red $(0.5 \%$ final concentration), and $1 \mathrm{nl}$ was pressure injected into one- to two-cell embryos using a Picospritzer II or an ASI MPPI-2 (Applied Scientific Instrumentation, Eugene, OR) injector. The injected volume was calculated using a calibrated eyepiece micrometer.

Cell transplants. For cell transplants, we used a heterochronic scheme (Moens and Fritz, 1999), diagrammed in Figure $5 a$, in which cells are taken from a donor at blastula stage ( $4 \mathrm{hpf}$ ), when cells are most easily removed, and transplanted into a host at gastrula stage ( $6 \mathrm{hpf}$ ), when the embryonic fate map is well established, with modifications from Moens and Fritz (1999). Donors were pressure injected at the one-cell stage with Alexa-568 dextran (Invitrogen), coinjecting 3 ng of DCC-MO1 for morphant donors. Morphant hosts were injected with $3 \mathrm{ng}$ of DCC-MO1. Donors were raised at RT to blastula stage, whereas hosts were raised at $28.5^{\circ} \mathrm{C}$ to shield stage. Embryos for transplants were mounted in individual wells in agarose plates (Kane and Kishimoto, 2002). Glass micropipettes were broken to a $\sim 30-40 \mu \mathrm{m}$ opening, beveled at $35^{\circ}$, and rinsed with hot water and EtOH between transplants. Cells from each donor were used to seed three hosts, targeting the junction between the margin and the shield. Host embryos were raised at $28.5^{\circ} \mathrm{C}$ in 24 -well plates coated with agarose.

When scoring transplant experiments, we selected chimeras in which GFP-positive cells populated either the presumptive REN (posterior r6) or CEN (r7) on one or both sides of the embryo (see Fig. 5). We only analyzed GFP-positive neurons that lay posterior to the horizontal line shown in Figure $5 c$ and were thus presumptive OLe neurons. We excluded those embryos in which there were Alexa-568-positive donor cells either surrounding or lateral to the GFP-positive cells, whose dendrites could potentially have served as scaffolds for the OLe dendrites.

Morphant phenotype analysis. All morpholino experiments were repeated at least twice. Confocal projections were scored according to four representative images of injected and wild-type embryos (live or fixed as appropriate), yielding a semiquantitative crossing score of $0-3$. Scoring was done by individuals blind to the experimental condition. For statis- 
tical purposes, classes $0 / 1$ and $2 / 3$ were combined, and Fisher's exact test was performed using InStat3 (GraphPad, San Diego, CA).

Microscopy and image analysis. Fixed or live embryos were embedded in 1.5\% low-melting agarose in $0.33 \times \mathrm{PBS}$ or E2/GN, respectively, dorsal down in a Petriperm dish (Greiner BioOne, Monroe, NC). Confocal $z$ stacks were acquired using an Olympus Fluoview confocal microscope. Fluoview or NIH ImageJ software was used to create confocal $z$ projections, and Adobe Photoshop and Adobe Illustrator (Adobe Systems, San Jose, CA) were used to merge images and compose figures. Because cell bodies were far brighter than processes in isl1: $g f p$ transgenics, gamma correction was used to make processes visible in Figures 3 and 4. We performed manual segmentation of OLe axons (see Fig. $3 e, f$ ) or faciomotor/octavolateralis efferent (nVII/OLe) neurons and their axons (see Fig. 6) by following the desired elements through all $z$ slices of the datasets and then coloring them green using Photoshop.

Cell transplant analysis. To generate the composite map of Figure $5 c$, confocal projections of individual WT $>$ WT transplants were overlaid in Adobe Illustrator on a reference image of an isl1:gfp transgenic. A red X was used to mark the center of each cell that clearly had a midlinecrossing process, and a blue $\mathrm{X}$ was used for each cell that clearly did not. A horizontal reference line was drawn to separate the majority of the red Xs from the majority of the blue Xs (see Fig. $5 c$ ). This line was overlaid on all cell transplant images, and only clones falling posterior to this line were analyzed. We excluded from analysis any chimeric embryos that had donor cells (Alexa-568 dextran-positive) intermixed in or lateral to the isll:gfp-positive CEN and REN clusters but included chimeras that only had donor cells in the midline (which is $d c c$ negative) or dorsal to the CEN/REN plane. For each rhombomere ( $\mathrm{r} 6$ or $\mathrm{r} 7$ ) containing isl1: $g f p$-positive donor cells, we noted whether there were (1) no dendrites, (2) dendrites that extended toward but failed to reach the midline, (3) dendrites that just reached the midline, or (4) dendrites that crossed the midline. For statistical purposes, classes $1 / 2$ and $3 / 4$ were combined, and Fisher's exact test was performed.

Statistical analysis. We used Fisher's exact test, a more restrictive form of contingency table analysis tests, to analyze our datasets, which were not large enough to make predictions for very large populations. In all cases, a two-tailed $p$ value was obtained using InStat3 (GraphPad).

\section{Results}

The isl1:gfp transgene labels the OLe neurons and their dendrites

The isl1:gfp transgenic line generated by the Okamoto laboratory (Higashijima et al., 2000) is a valuable tool for visualizing the cranial motor neurons, including OLe neurons. At $36 \mathrm{hpf}$, this transgene labels clusters of REN and CEN neurons intermingled with facial motor neurons in rhombomeres $\mathrm{r} 6$ and $\mathrm{r} 7$, and fine processes can be seen crossing the midline in these rhombomeres (Higashijima et al., 2000) (Fig. 1a,d,h). These processes have been interpreted to be the contralateral dendrites of OLe neurons (Higashijima et al., 2000), because HRP back-labeling from the posterior lateral line had shown
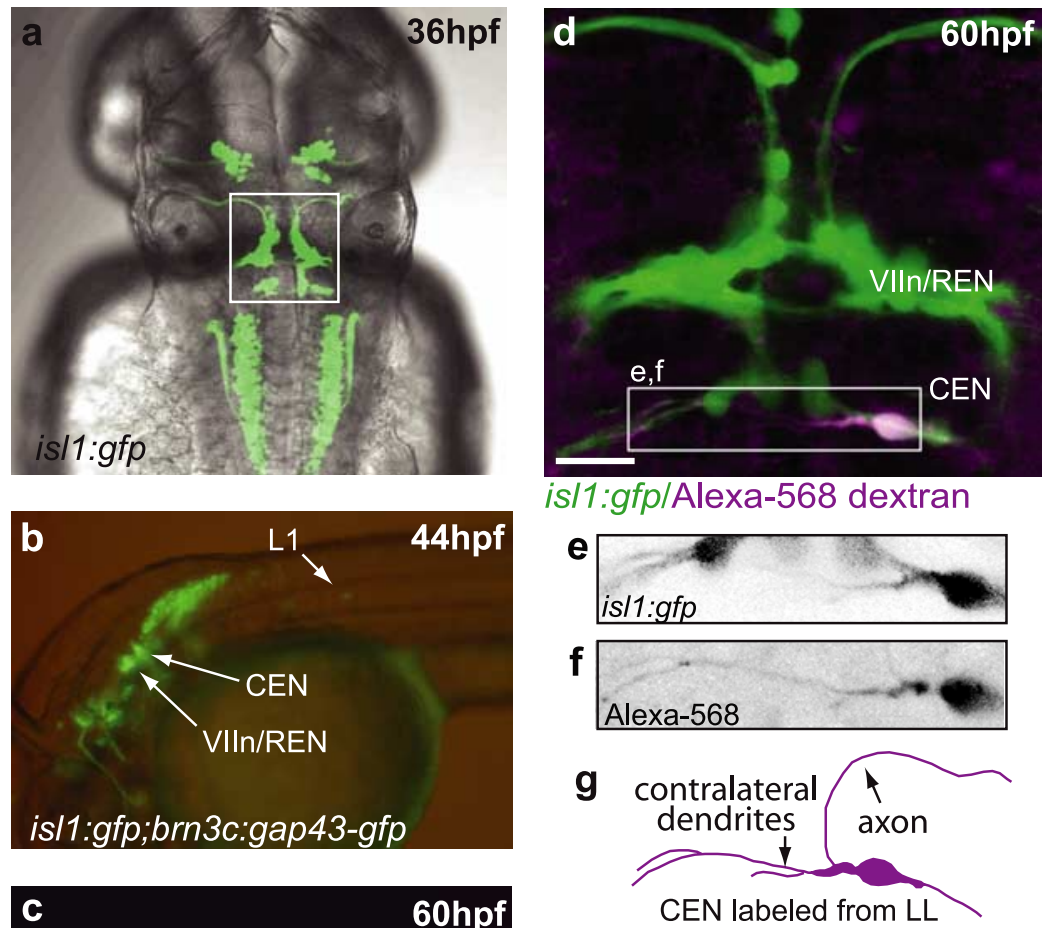

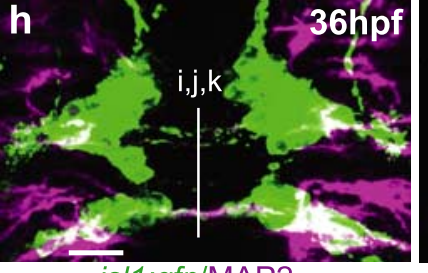

is/1:gfp/MAP2

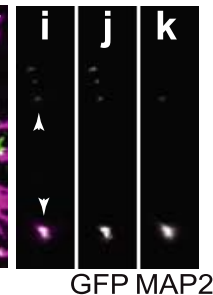

GFP MAP2
Figure 1. The is/1:gfp transgene labels 0 Le neurons and their contralateral dendrites. Alexa-568 dextran (magenta) injected in the lateral line at the L1 neuromast level retrogradely labeled a CEN neuron and its dendrites. $\boldsymbol{a}$, Dorsal view of is 11:gfp embryo ( $\mathrm{hpf}$ ) in which the L1 neuromast is labeled by brn3c:gap43-gfp. c, Lateral image of embryo at $60 \mathrm{hpf}$ showing the uptake of (is area at higher magnification show colocalization of is/1:gfp (e) and Alexa-568 dextran $(\boldsymbol{f})$, reversed contrast. (he the labeled CEN neuron from d, traced from the threenal dataset. $h$, MAP2 staining -dimensional dataset from $\boldsymbol{h}$ at level of vertical line shows colocalization (white arrowheads) of GFP $(\boldsymbol{j})$ and MAP2 $(\boldsymbol{k})$ in cross section. Some GFP-positive contralateral projections do not display MAP2 staining, perhaps attributable to dendrite immaturity. Scale bars, $20 \mu \mathrm{m}$.

previously that OLe neurons bear contralateral dendrites (Metcalfe et al., 1985; Bricaud et al., 2001).

To test directly whether these midline processes are indeed OLe dendrites, we repeated the back-label experiments in the isl1:gfp line, injecting Alexa-568 dextran into the posterior lateral line nerve at the level of the L1 neuromast in $44 \mathrm{hpf}$ embryos (Fig. $1 b)$. To visualize the L1 neuromast, we used embryos from an outcross of the isl1:gfp line with the $\operatorname{Tg}(\text { brn } 3 c \text { : } g a p 43-g f p)^{\text {s356t }}$ line in which the neuromasts are GFP labeled (Xiao et al., 2005). By 60 hpf, the dye was taken up by OLe axons projecting to the posterior lateral line (Fig. 1c) and retrogradely labeled CEN neurons and their dendrites (Fig. $1 d-g$ ). Of six CEN neurons back-labeled in five embryos, all were GFP positive and had contralaterally projecting dendrites, showing that at least some of the isll:gfppositive midline processes are indeed OLe dendrites. This conclusion is also supported by our cell transplant data (see Fig. $\left.6 b, c, c^{\prime}\right)$. A similar result has been obtained recently with DiI la- 

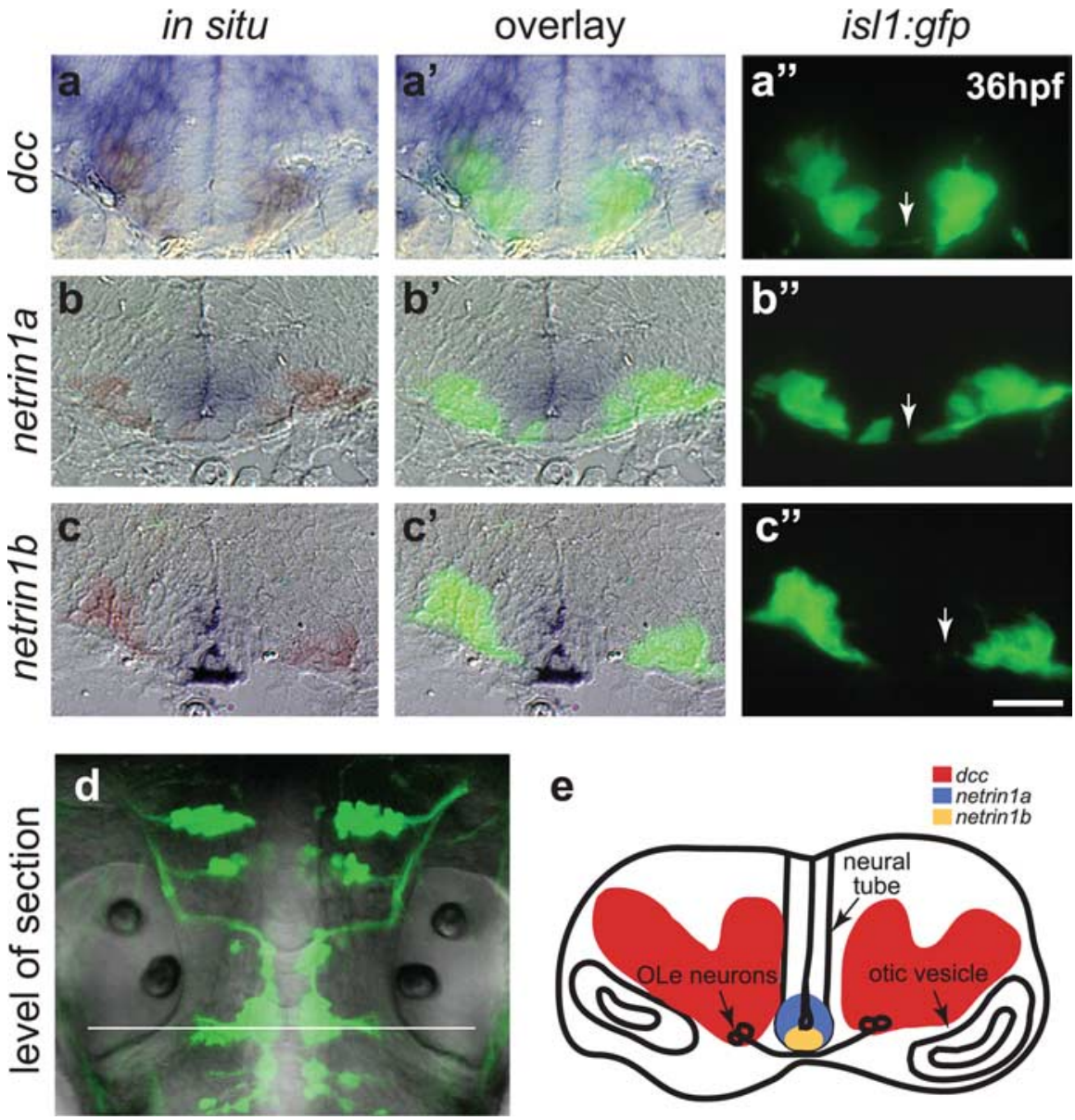

Figure 2. dcc is expressed in 0 Le neurons, whereas netrin $1 a$ and netrin $1 b$ are expressed at the midline at 36 hpf. $\boldsymbol{a}-\boldsymbol{c}^{\prime \prime}$, Sections through hindbrain at the level shown in $\boldsymbol{d}$; dorsal is up, and ventral is down. $\boldsymbol{a}-\boldsymbol{c}$, Bright-field images showing expression of $d c c$, netrin 1a, and netrin 1b. Purple is in situ hybridization reaction product; orange is Alexa-488 tyramide labeling is/1:gfp cells. $\boldsymbol{a}^{\prime \prime}-\boldsymbol{c} \boldsymbol{c}^{\prime \prime}$, Fluorescent images of is/1:gfp-positive cells, comprising OLe and VII neurons. Arrows indicate position of midline-crossing dendrites. $\boldsymbol{a}^{\prime}-\boldsymbol{c}^{\prime}$, Merged images. $\boldsymbol{d}$, Level of section in $\boldsymbol{a}-\boldsymbol{c}$ shown on a confocal projection of an is/1:gfp embryo. Rostral is up. $\boldsymbol{e}$, Summary of gene expression. Scale bar, $50 \mu \mathrm{m}$.

beling (Sapede et al., 2005). To further confirm the identity of these midline-crossing processes, we double-stained isl1:gfp embryos for the dendrite marker MAP2 (Fig. 1h-k) (Tomasiewicz and Wood, 1999). We find that the majority of GFP-positive projections are MAP2 positive, confirming their dendritic identity.

$d c c$ is expressed in the OLe neurons, whereas netrin 1 genes are expressed at the midline

To test whether Netrin/DCC signaling might guide OLe dendrites, we first determined whether $d c c$ and netrin $1 a$ and netrin $1 b$, the gene-duplicated zebrafish orthologs of mammalian netrin1, are expressed appropriately. Although previous work had shown that netrin $1 a$ and netrin $1 b$ are expressed in the midline (Lauderdale et al., 1997; Strahle et al., 1997), their expression in the hindbrain had not been described precisely. We performed in situ hybridization in isl1:gfp embryos to establish the expression of $d c c$, netrin $1 a$, and netrin $1 b$ in relationship to OLe neurons, by sectioning the hindbrain at levels that included the OLe dendrites. At $36 \mathrm{hpf}$, shortly after OLe contralateral dendrites first cross the midline, $d c c$ is expressed in a broad domain that appears to include all isl1:gfp neurons (and thus the OLe neurons) but excludes the floor plate and the ventricular cells along the dorsoventral extent of the midline (Fig. $2 a-a^{\prime \prime}$ ). netrin $1 a$ is expressed in the ventralmost neural tube (Fig. $2 b-b^{\prime \prime}$ ), whereas netrin $1 b$ is expressed in the floor plate (Fig. $2 c-c^{\prime \prime}$ ). Note that netrin 2 and netrin4, the two other known secreted netrin genes in zebrafish, are not expressed in the ventral hindbrain at this stage (Park et al., 2005). These expression patterns show that OLe neurons (and presumably their dendrites) express the DCC receptor as their dendrites grow toward the midline, which is a source of Netrin1.

\section{OLe dendrites fail to cross the midline after knockdown of $d c c$}

To test the requirement of $d c c$ in OLe dendrite guidance, we designed three antisense MOs and injected them into isll:gfp embryos at the one- to two-cell stage to knock down DCC. In embryos injected with DCC-MO1, which binds at the start codon of the $d c c$ mRNA, OLe contralateral dendrites either completely failed to cross at the midline or were significantly fewer in number in embryos fixed at $36 \mathrm{hpf}$ (Fig. $\left.3 c, c^{\prime}\right)$. This effect was not seen with a standard control MO at the same dose (Fig. $\left.3 b, b^{\prime}\right)$. Because we could not resolve single dendrites even with confocal microscopy, we adopted a semiquantitative crossing score, assigning embryo to classes $0-3$ by comparison with four representative images of injected and wild-type embryos (Fig. 3d). Whereas 25 of 28 control morphants (MO-injected embryos) fell in classes 2 and 3, with many midlinecrossing dendrites closely resembling wild-type (24 of 27 embryos), the DCCMO1 morphants all (27 of 27) fell into classes 0 and 1 , representing few or no crossing dendrites (Fig. $3 c^{\prime}$, Table 1). For statistical purposes, classes 0 and 1 were pooled, as were classes 2 and 3 (Table 1). DCC-MO1 morphants showed significantly less crossing than wild type $(p<0.0001)$, whereas control morphants were not significantly different from wild-type $(p=1.0)$. The lack of midline crossing by the OLe contralateral dendrites may either reflect a failure in dendrite formation or a failure in guidance toward the netrin1a-expressing midline.

d cc knockdown does not affect OLe cell fate or axon guidance Might the reduced crossings reflect an overall delay in development of the morphants? We believe not, for four reasons. First, the overall morphology of DCC-MO1 morphants was grossly similar to wild-type and control morphants (data not shown). Second, the development of the retinotectal projection at 48 and $72 \mathrm{hpf}$ was indistinguishable between morphants and wild-types (data not shown). Third, the morphology of the isll:gfp-positive clusters in morphants was very similar to wild type at $36 \mathrm{hpf}$. Because these neurons migrate caudally during development, only reaching their positions in $\mathrm{r} 6$ and $\mathrm{r} 7$ between 30 and $36 \mathrm{hpf}$ (data not shown) (Higashijima et al., 2000), even a slight retardation would be expected to affect cluster morphology. Finally, reduced crossing is seen even when DCC morphant cells are transplanted into wild-type hosts (see Fig. 5 and Table 3). 

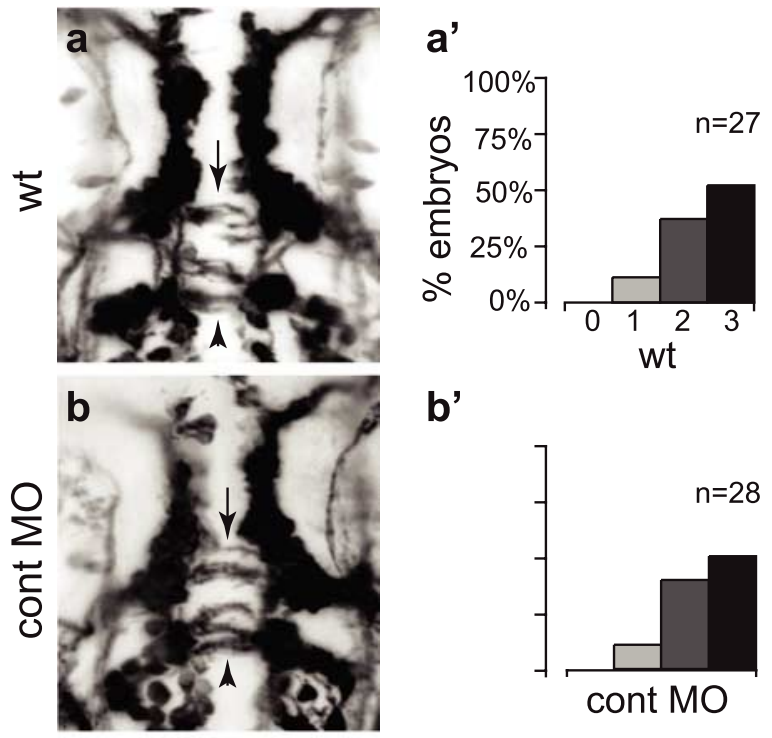

b'
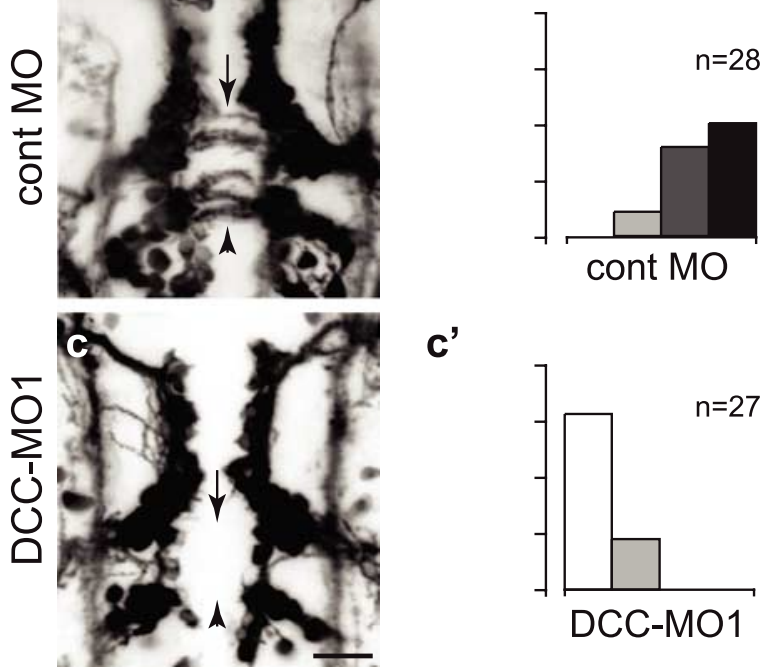

C'
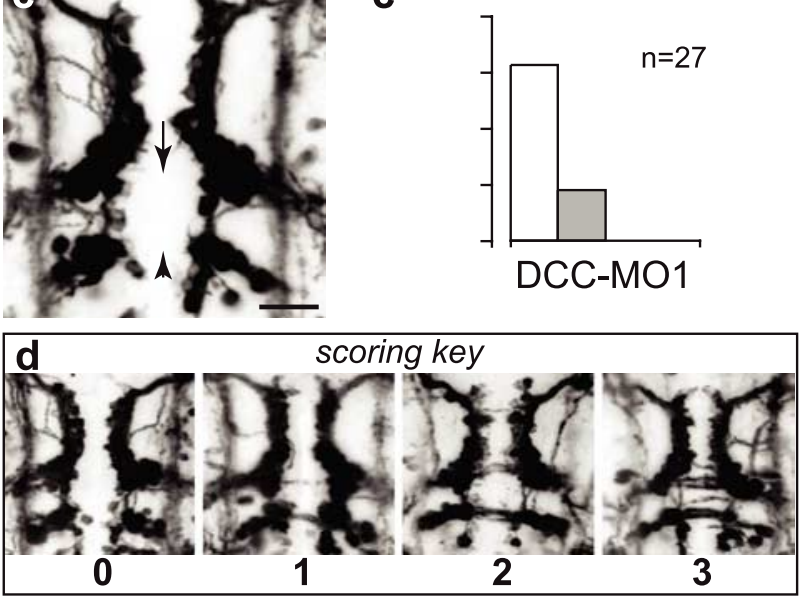

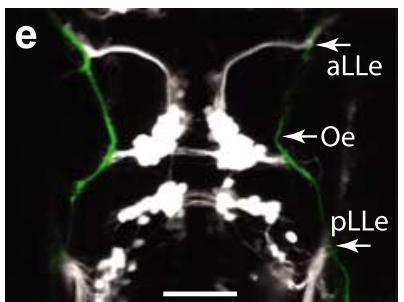

wt

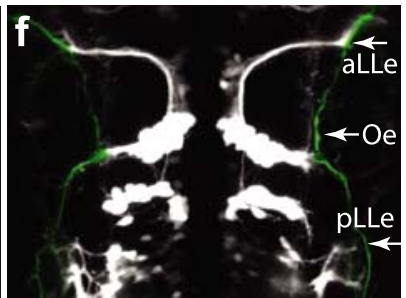

DCC-MO1
Figure 3. OLe contralateral dendrites are missing or fail to cross the midline after knockdown of DCC by DCC-M01. $\boldsymbol{a}-\boldsymbol{d}$, Confocal projections of hindbrain at the level of OLe and VII neurons. Anti-GFP immunostaining of is/1:gfp transgene in fixed $36 \mathrm{hpf}$ embryos, reversed contrast. Rostral is up, and caudal is down. Normal positions of contralateral dendrites indicated by arrow (REN) or arrowhead (CEN). $\boldsymbol{d}$, Key used to score fixed embryos as classes 0 (no crossing) to 3 (full crossing) for $\boldsymbol{a}^{\prime}-\boldsymbol{c}^{\prime} \cdot \boldsymbol{a}, \boldsymbol{a}^{\prime}$, WT embryos have normal crossings and fall mostly in classes 2 and $3 . \boldsymbol{b}, \boldsymbol{b}^{\prime}$, OLe contralateral dendrites are unaffected after injection of $3 \mathrm{ng}$ of control MO. $\boldsymbol{c}$, $\boldsymbol{c}^{\prime}$, OLe contralateral dendrites are greatly reduced after injection of $3 \mathrm{ng}$ of DCC-M01.e, $\boldsymbol{f}$, Confocal projections of live is/1:gfp WT embryos (e) and DCC - M01 morphants $(\boldsymbol{f})$ at $36 \mathrm{hpf}$. OLe, $\mathrm{VII}$, and $\mathrm{X}$ neurons and their projections are shown in white, whereas 0 Le axonal projections, traced in the full three-dimensional dataset, are pseudocolored in green. Morphants show normal aLLe, pLLe, and 0 e, indicating that 0 Le neurons are correctly specified. Scale bars: $\boldsymbol{a}-\boldsymbol{c}$, $25 \mu \mathrm{m} ; \boldsymbol{e}, \boldsymbol{f}, 50 \mu \mathrm{m}$.

Could the neurons fail to put out contralateral dendrites because they are misspecified, for instance adopting a branchiomotor neuron rather than an OLe fate? Because we know of no molecular markers that distinguish OLe neurons from other cra-
Table 1. OLe dendrite crossing is unaffected by control $\mathrm{MO}$ and strongly reduced by DCC-M01

\begin{tabular}{llll}
\hline & \multicolumn{2}{l}{ Number of embryos } \\
\cline { 2 - 4 } & Classes 0 and 1 & Classes 2 and 3 & $\begin{array}{l}\text { Two-tailed } p \text { value } \\
\text { (Fisher's exact test) }^{a}\end{array}$ \\
\hline WT & 3 & 24 & \\
Control M0 (3 ng) & 3 & 25 & $p=1.0$ \\
DCC-M01 (3 ng) & 27 & 0 & $p<0.0001$ \\
\hline
\end{tabular}

Embryos were scored as shown in Figure 3.

${ }^{a}$ Fisher's exact test, morphants compared with WT.

nial motor neurons, we instead analyzed their axonal projections and found them to be normal in morphants. In class $0-1$ morphants, 13 of 13 embryos had anterior lateral line efferents (aLLe), 17 of 17 had octaval efferents (Oe), and 17 of 17 had posterior lateral line efferents (pLLe) (Fig. $3 e, f$ ). The thickness of these efferent axon bundles was indistinguishable between WT and morphants. The presence of properly directed OLe efferent axons in the DCC-MO1 morphants shows that at least some, and indeed likely all, OLe neurons retain their identity and continue to relay efferent information to the lateral line and ear. Thus, Netrin/DCC signaling acts primarily in the OLe contralateral dendrites, without evident effects on cell fate or axon guidance.

To confirm that the OLe dendrite phenotype is specific to the loss of $d c c$ function and rule out a nonspecific effect of DCCMO1 on some other gene, we designed two more DCC MOs. DCC-MO2 is a translation-blocking MO that binds the dcc 5' UTR 42 bp upstream from DCC-MO1. Both DCC-MO1 and DCC-MO2 are able to prevent translation of injected mRNAs encoding GFP fused downstream of the corresponding target sequence (M. Bak-Maier and S. Fraser, personal communication). isl1:gfp embryos injected with DCC-MO2 were scored as before, except that they were imaged live at $36 \mathrm{hpf}$, and again showed reduced dendrite crossing ( 0 of 28 in classes 2 or 3 ) relative to wild-type (26 of 27; $p<0.0001$ ), albeit at slightly higher dose than DCC-MO1 (Fig. $4 b, b^{\prime}$, Table 2).

Finally, DCC-MO3 is a splice-blocking MO that binds to the first splice donor after the dcc start codon. Although DCC-MO3 efficiently blocks normal splicing of the first intron at a dose of 2 ng (supplemental Fig. 1, available at www.jneurosci.org as supplemental material), reverse transcription-PCR and sequencing of the resulting misspliced product revealed that a cryptic splice donor in the intron leads to an in-frame insertion of 24 amino acids immediately after the DCC signal sequence. Perhaps because this mutant protein is partly functional, disruption of dendrite crossing by DCC-MO3 was incomplete ( 2 of 29 in class 2/3), although significant compared with wild type (26 of $27 ; p<$ 0.0001 ) (Fig. $4 c, c^{\prime}$, Table 2).

Although it would have been valuable to directly assay the level of DCC protein knockdown, three different commercial anti-DCC antibodies failed to cross-react to zebrafish. However, the concordance of results from three independent MOs of confirmed efficacy is strong evidence that this phenotype is indeed specific to the knockdown of $d c c$ and that $d c c$ is required for OLe dendrites to cross the midline.

OLe dendrites fail to cross the midline after knockdown of netrin $1 a$ and netrin $1 b$

If Netrin 1/DCC signaling is indeed required for OLe dendrites to grow toward the midline, then knocking down netrin 1 should reproduce the $d c c$ knockdown phenotype. To test this hypothesis, we knocked down both zebrafish netrin1 genes by coinjecting a 


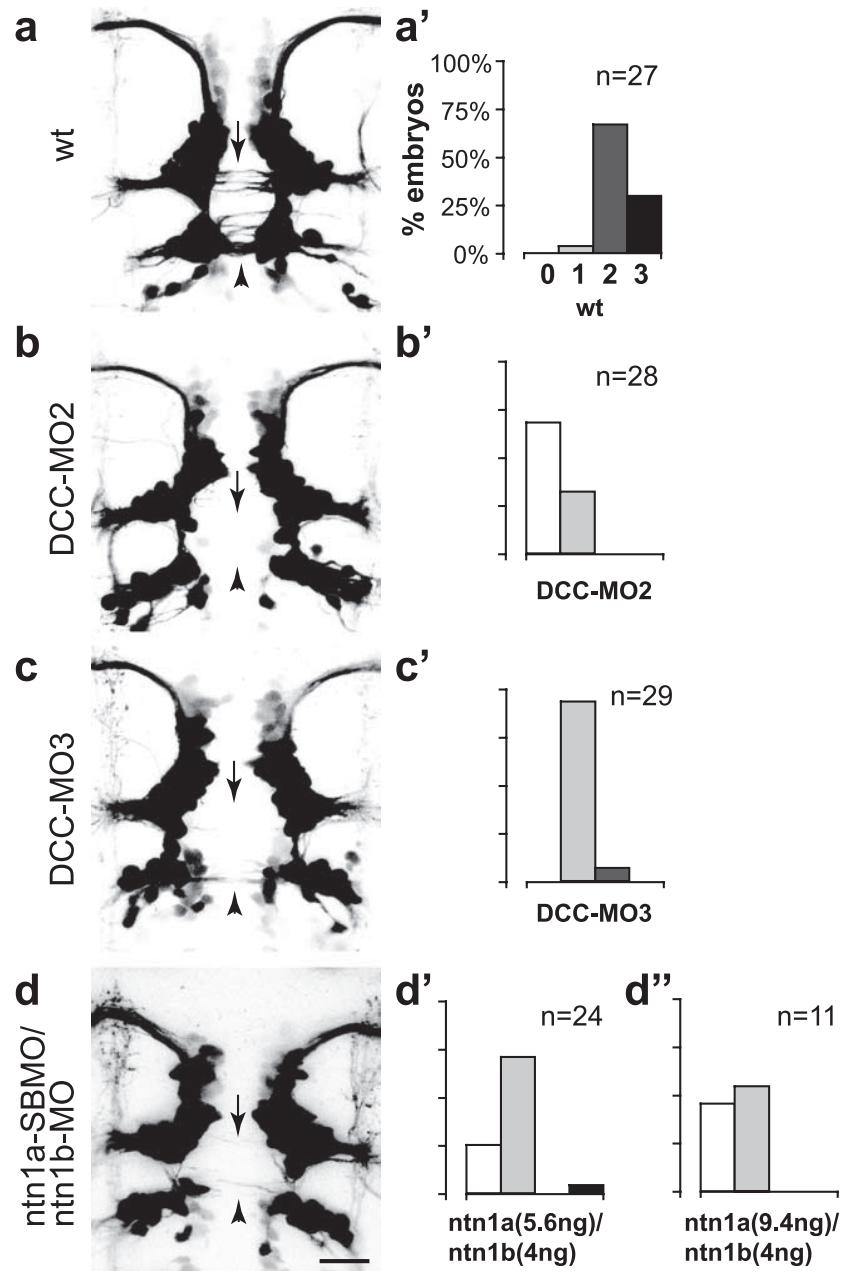

e

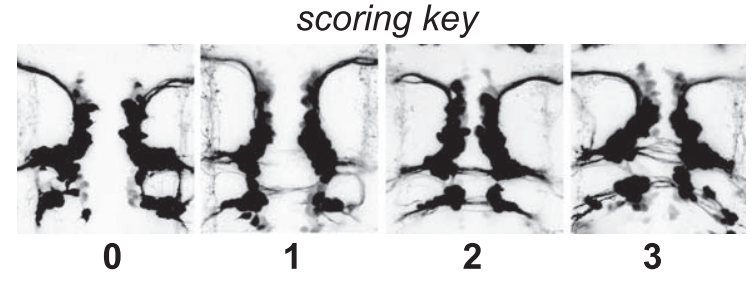

Figure 4. OLe contralateral dendrites are missing or fail to cross the midline after knockdown of DCC by DCC $-\mathrm{M02}$ or DCC - M03 or knockdown of Netrin 1 by coinjection of netrin1a splice-blocking M0 (ntn1a-SBM0) and netrin1b-MO (ntn1b-M0). $a-f$, Confocal projections of the hindbrain of live $36 \mathrm{hpf}$ embryos, reversed contrast. Rostral is up, and caudal is down. Normal positions of contralateral dendrites indicated by arrow (REN) or arrowhead (CEN). $a, a^{\prime}$, WT embryos show normal midline crossing by OLe dendrites. $\boldsymbol{b}, \boldsymbol{b}^{\prime}, \boldsymbol{c}, \boldsymbol{c}^{\prime}, 0$ Le dendrites are strongly reduced by DCC $-\mathrm{MO2}(5 \mathrm{ng})$ and DCC $-\mathrm{M} 03(2 \mathrm{ng}) . \boldsymbol{d}, \boldsymbol{d}^{\prime}, 0$ Le dendrites are strongly reduced after knocking down both netrin $1 a$ and netrin $1 b$ by coinjection of netrin 1a-SBM0 ( 5.6 $\mathrm{ng}) /$ netrin1b-M0 (4 ng) or netrin1a-SBM0 (9.4 ng)/netrin1b-M0 (4 ng). e, Scoring key for $\boldsymbol{a}^{\prime}-\boldsymbol{d}^{\prime \prime}$ (live embryos). Scale bar, $25 \mu \mathrm{m}$.

Table 2. OLe dendrite crossing is strongly reduced by DCC $-\mathrm{MO2}$, DCC $-\mathrm{MO3}$, and netrin $\mathrm{MO}$ coinjection

\begin{tabular}{llll}
\hline & Number of embry0s & \\
\cline { 2 - 4 } & Classes 0 and 1 & Classes 2 and 3 & $\begin{array}{c}\text { Two-tailed } p \text { value } \\
\text { (Fisher's exact test) }\end{array}$ \\
\hline WT & 1 & 26 & \\
DCC-M02 (5 ng) & 28 & 0 & $p<0.0001$ \\
DCC-M03 (2 ng) & 27 & 2 & $p<0.0001$ \\
netrin1a-SBM0 (5.6 ng)/netrin1b-M0 (4 ng) & 23 & 1 & $p<0.0001$ \\
netrin1a-SBM0 (9.4 ng)/netrin1b-M0 (4 ng) & 11 & 0 & $p<0.0001$ \\
\hline
\end{tabular}

Embryos were scored as shown in Figure 4.

${ }^{a}$ Fisher's exact test, morphants compared with WT.
Table 3. DCC function is necessary and sufficient in transplanted cells

\begin{tabular}{|c|c|c|c|c|}
\hline & donor $>$ host & reaching or crossing & absent or extending & $\begin{array}{c}\text { Two-tailed } \\
\text { p-value } \\
\text { (Fisher's } \\
\text { exact test)* }\end{array}$ \\
\hline \multirow{3}{*}{ 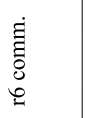 } & $w t>w t$ & $21(84 \%)$ & $4(16 \%)$ & \\
\hline & $w t>$ DCC MO1 & $9(69 \%)$ & $4(31 \%)$ & 0.41 \\
\hline & DCC MO1>wt & $4(17 \%)$ & $19(83 \%)$ & $<0.0001$ \\
\hline \multirow{3}{*}{ 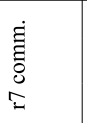 } & $\mathrm{wt}>\mathrm{wt}$ & $7(78 \%)$ & $2(22 \%)$ & \\
\hline & wt $>$ DCC MO1 & $12(92 \%)$ & $1(8 \%)$ & 0.54 \\
\hline & DCC MO1>wt & $4(27 \%)$ & $11(73 \%)$ & 0.03 \\
\hline \multirow{3}{*}{ 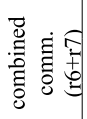 } & $w t>w t$ & $28(82 \%)$ & $6(18 \%)$ & \\
\hline & wt $>$ DCC MO1 & $21(81 \%)$ & $5(19 \%)$ & 1.0 \\
\hline & DCC MO1>wt & $8(21 \%)$ & $30(79 \%)$ & $<0.0001$ \\
\hline
\end{tabular}

Rhombomeres containing transplanted isll:gfp cells were scored for commissures, grouping those that had no commissures or did not reach the midline and those that reached or crossed the midline. *Fisher's exact test, comparing to WT > WT transplants.

splice-blocking $\mathrm{MO}$ against netrin1a (netrin1a-SBMO) (for confirmation of efficacy, see supplemental Fig. 2, available at www. jneurosci.org as supplemental material) and a translationblocking MO against netrin $1 b$ (netrin1b-MO). Indeed, this led to embryos with reduced or absent OLe dendrites at the midline (Fig. $4 d, d^{\prime}$, Table 2) ( 23 of 24 in class $0 / 1$ compared with 1 of 27 in WT; $p<0.0001)$. Furthermore, increasing the dose of netrin 1 SBMO led to more severe phenotypes (Fig. $4 d^{\prime \prime}$, Table 2). Interestingly, dendrites usually crossed normally when either netrinlaSBMO or netrin $1 \mathrm{~b}-\mathrm{MO}$ was injected individually (data not shown). This suggests that either netrin 1 gene alone is sufficient to attract the dendrites at the midline. Because knocking down either ligand or receptor yields the same phenotype, we conclude that Netrin1/DCC signaling is necessary for OLe dendrites.

\section{DCC acts cell autonomously in OLe dendrites}

Does DCC act directly in growing OLe dendrites to attract them toward the midline, or alternately, might DCC guide earlier axons or dendrites, which then act as a scaffold along which the OLe dendrites grow? To distinguish between these possibilities, we performed cell transplants between $d c c$ morphants and wild-type embryos. If DCC acts cell autonomously, transplanted morphant OLe neurons should not form contralateral dendrites in a WT host, whereas transplanted WT OLe neurons should be able to put forth contralateral dendrites even in a host lacking DCC protein.

As diagrammed in Figure $5 a$, isl1:gfp donor cells labeled with the cell-lineage marker Alexa-568 dextran were transplanted into nontransgenic hosts. Transplanting WT isll:gfp cells into WT hosts allowed us to visualize individual OLe neurons (defined by the presence of contralateral dendrites) and to compile a composite map (Fig. $5 c$ ) of their cell body positions relative to those of facial motor neurons (GFP-positive neurons in $\mathrm{r} 6$ and r7 without contralateral dendrites) and draw a line (Fig. $5 c$ ) that primarily separated the facial motor neurons from the OLe neurons. We analyzed each rhombomere ( $\mathrm{r} 6$ or $\mathrm{r} 7$ ) that contained isl1:gfp neurons lying posterior to this line (i.e., presumptive donor OLe neurons) and scored GFP-positive dendritic commissures, on a scale of "absent," "extending," 
"reaching midline," or "crossing midline" (Table 3). Results for r6 and r7 were combined, because they were statistically indistinguishable.

When we transplanted cells from DCC-MO1 morphants into WT, only 8 of 38 (21\%) rhombomeres had commissures that reached or crossed the midline (Fig. $5 f-f^{\prime \prime}$, Table 3$)$, significantly fewer than in WT $>$ WT controls ( 28 of 34 or $82 \%$; $p<$ 0.0001 ) (Fig. $5 d-d^{\prime \prime}$, Table 3 ). The occasional contralateral dendrites in morphant $>$ WT transplants likely reflect incomplete knockdown by DCC-MO1 (Fig. $3 c-c^{\prime \prime}$, Table 1), whereas the occasional missing dendrites in WT $>$ WT transplants likely reflects a subset of OLe neurons that are still in the process of extending contralateral dendrites and possibly also a set of isl1:gfp neurons that do not have contralateral dendrites (perhaps intermingled facial motor neurons). Conversely, when we transplanted WT cells into DCC-MO1 morphants, we found a very similar incidence of midline reaching/ crossing as in WT $>$ WT $(21$ of 26 or $81 \%$; $p=1.0$ ) (Fig. 5e-e', Table 3). From these results, we conclude that DCC indeed acts cell autonomously for dendrite crossing, being necessary and sufficient in the OLe neurons but not their environment.

Furthermore, WT $>$ WT transplants with few donor cells showed beautifully the normal morphology of single isll:gfp cells. In five of six such embryos, we clearly saw that individual neurons with contralateral dendrites sent axons into the aLLe nerve, consisting of supraorbital and infraorbital lateral line efferents (Fig. $\left.6 b, c, c^{\prime}\right)$. Together with the back-labeling of Figure 1, this supports the conclusion that isll:gfp neurons with contralateral dendrites are indeed OLe neurons.

To analyze cell fate and axon guidance of individual morphant cells in a wild-type background, we looked at DCC-MO1 morphant $>$ WT transplants with no more than five transplanted cells in the REN or CEN. In four of five WT hosts, transplanted morphant cells with no contralateral dendrites sent axons to innervate at least one of the OLe targets: the aLL, pLL, or ear (Fig. $6 d, e, e^{\prime}$ ). As expected, more than one OLe target was innervated in embryos with more transplanted cells (data not shown). These results were very similar to $\mathrm{WT}>\mathrm{WT}$ transplants (Fig. $6 b, c, c^{\prime}$ ) and are significant for three reasons. First, they give us additional confidence that OLe neurons retain their identity in the absence of Netrin/ DCC signaling. Second, the fact that transplanted morphant cells with normal OLe a

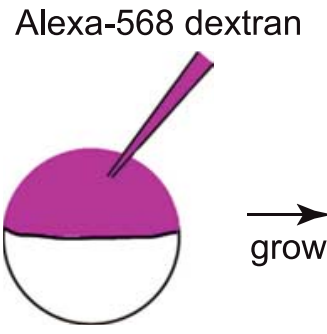

is/1:gfp donor 1 cell stage heterochronic

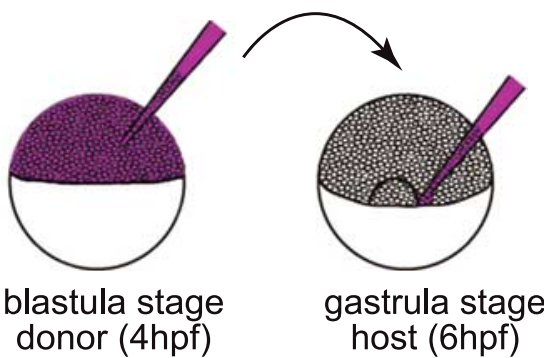
transplant
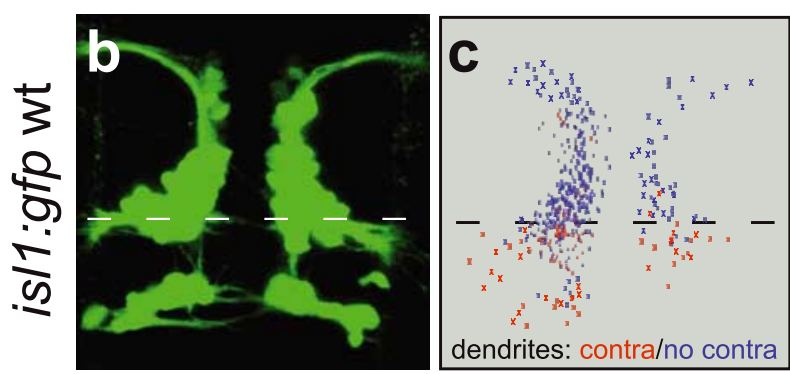

$w t=w t$
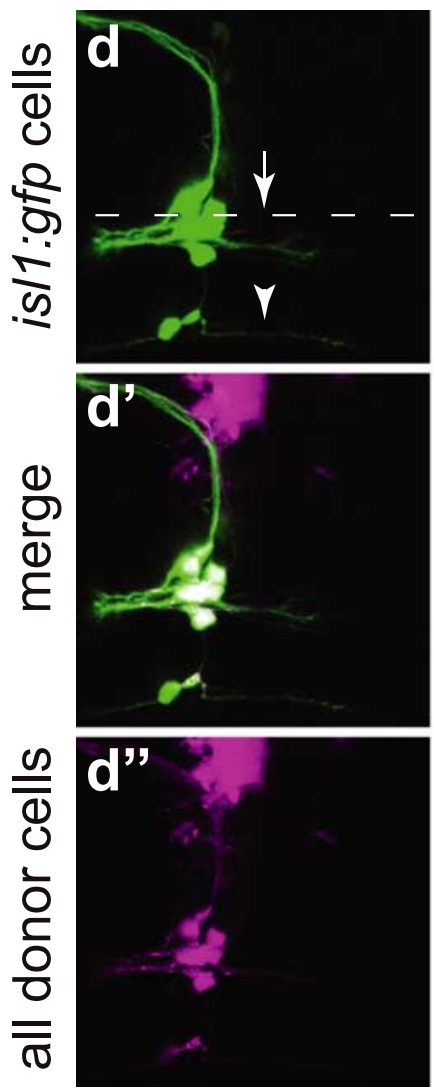

wt - DCC-MO1
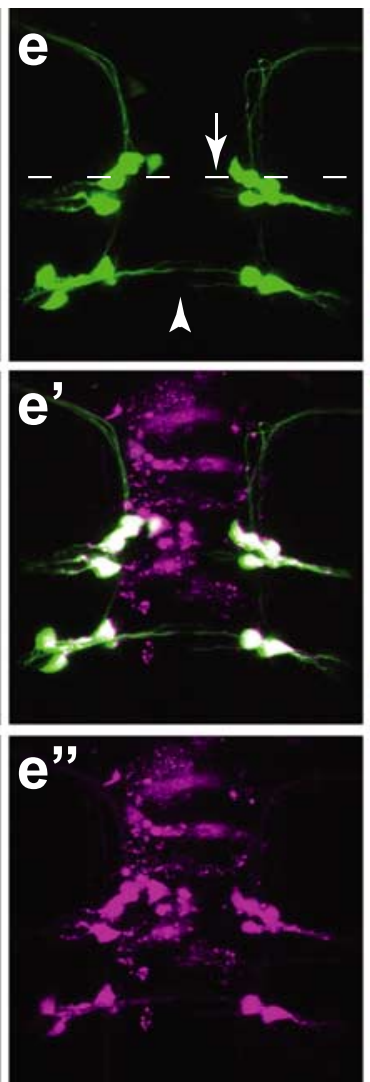

$\mathrm{DCC}-\mathrm{MO} 1-\mathrm{wt}$
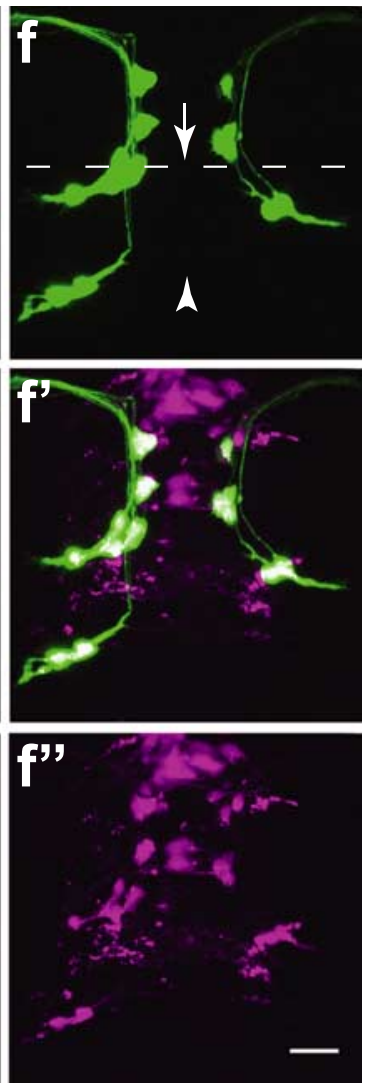

Figure 5. DCC acts cell autonomously in OLe neurons. $\boldsymbol{a}$, Schematic of cell transplants. $\boldsymbol{b}$, Reference image of WT is/1:gfp embryo at $36 \mathrm{hpf}$ (z-stack confocal projection). c, Composite map of cell bodies with contralateral dendrites (presumptive 0Le, red) and without contralateral dendrites (presumptive facial motor neurons, blue). The horizontal reference line divides the majority of facial neurons from the majority of OLe neurons; only cells lying posterior of this line were analyzed in transplants (see Materials and Methods). $\boldsymbol{d}-\boldsymbol{f}^{\prime}$, Confocal projections of live 36 hpf embryos, showing transplanted is/1:gfp cells (d-f), Alexa-568 dextran (magenta, all donor cells; $\boldsymbol{d}^{\prime \prime}-\boldsymbol{f}^{\prime}$ ), and merged images (colocalization shown in white) $\left(\boldsymbol{d}^{\prime}-\boldsymbol{f}^{\prime}\right)$. Arrows, Position of REN dendrites; arrowheads, position of CEN dendrites. Rostral is up, and caudal is down. $\boldsymbol{d}-\boldsymbol{d}^{\prime \prime}$, In controls, WT OLe neurons transplanted into a WT host have dendrites that cross the midline. $\boldsymbol{e}-\boldsymbol{e}^{\prime \prime}$, WT OLe neurons transplanted into a DCC morphant also have dendrites that cross the midline. $f-f^{\prime}$, DCC-deficient OLe neurons transplanted into a WT host lack midline-crossing dendrites. Scale bar, $20 \mu \mathrm{m}$. 
a
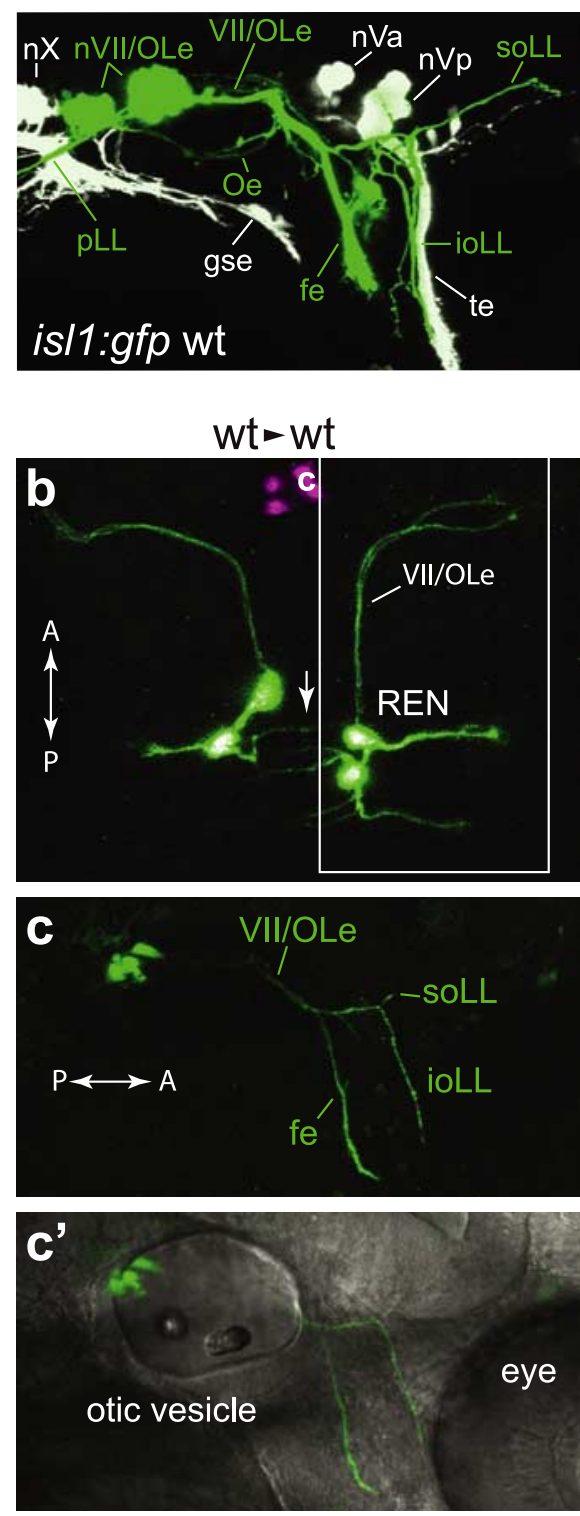
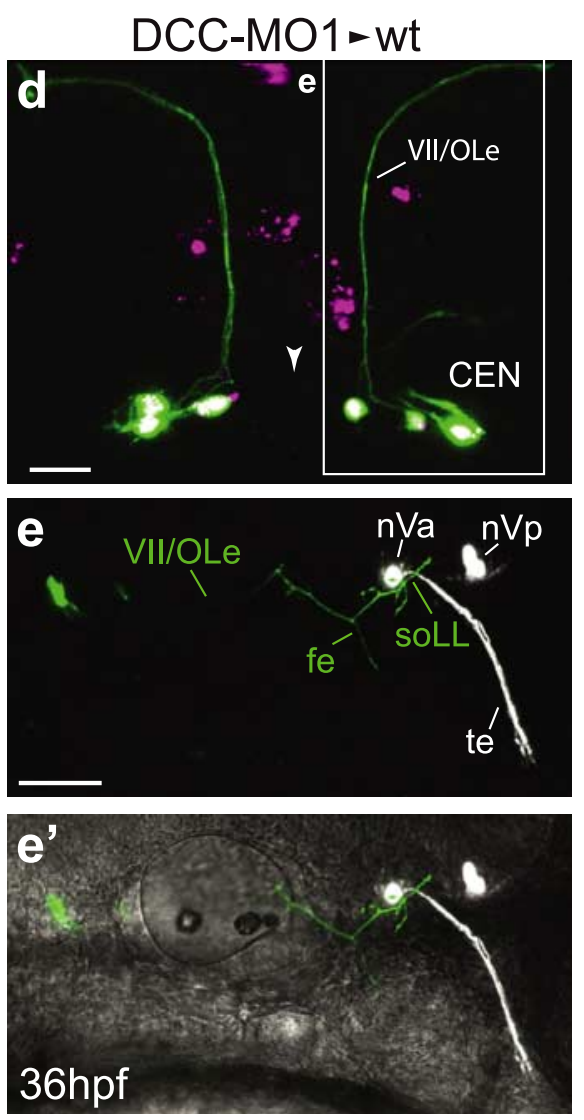

Figure 6. DCC-deficient OLe neurons innervate correct targets. a, Lateral view of WT is/1:gfp embryo at 36 hpf to show VII/OLe efferent projections in their normal setting. nVII and nOLe cell bodies and their projections are highlighted in green. Nuclei: nX, vagus; nVII/OLe, faciomotor/octavolateral; $\mathrm{nVa}$, anterior trigeminal; $\mathrm{nVp}$, posterior trigeminal. Efferents: VII/OLe, faciomotor/octavolateral; fe, faciomotor; gse, glossopharyngeal; soLL, supraorbital lateral line, ioLL, infraorbital lateral line; te, trigeminal. $\boldsymbol{b}-\boldsymbol{e}^{\prime}$, Confocal projections of a WT $>$ WT chimera $\left(\boldsymbol{b}-\boldsymbol{c}^{\prime}\right)$ and a DCC - M01 $>$ WT chimera $\left(\boldsymbol{d}-\boldsymbol{e}^{\prime}\right)$, imaged live at $36 \mathrm{hpf}$. $\boldsymbol{b}, \boldsymbol{d}$, Dorsal views, showing GFP (green) merged with Alexa-568 dextran (magenta) (colocalization is shown in white). $\boldsymbol{c}, \boldsymbol{c}^{\prime}, \boldsymbol{e}, \boldsymbol{e}^{\prime}$, The right halves of the same two embryos, reimaged in a lateral view extending slightly anterior of boxes in $\boldsymbol{B}$ and $\boldsymbol{D} \cdot \boldsymbol{e}, \boldsymbol{e}^{\prime}, \mathrm{nVII} / 0 \mathrm{Le}$ (faciomotor and octavolateral) neurons highlighted in green; other is11:gfp-positive neurons are in white. A, Anterior; $P$, posterior. $C^{\prime}, e^{\prime}$, GFP fluorescence overlaid on bright-field image to show otic vesicle and eye as landmarks. $\boldsymbol{b}, \boldsymbol{c}, \boldsymbol{c}^{\prime}$, In this WT $>$ WT chimera, only a few REN neurons were transplanted. They bear contralateral dendrites $\left(\boldsymbol{b}\right.$, arrow) and send axons to the supraorbital and infraorbital lateral line (soLL, ioLL; $\left.\boldsymbol{c}, \boldsymbol{c}^{\prime}\right)$, which are part of the anterior lateral line. A facial efferent (fe) is labeled, indicating that the transplanted cells include a faciomotor neuron. $\boldsymbol{d}, \boldsymbol{e}, \boldsymbol{e}^{\prime}$, In this DCC $-\mathrm{M} 01>$ WT chimera, a few CEN neurons were transplanted. They all lack contralateral dendrites (d, arrowhead) but bear ipsilateral dendrites and project axons to the supraorbital lateral line $(\boldsymbol{e})$. An axon from a transplanted faciomotor neuron also enters the facial efferent nerve. Transplanted neurons of the trigeminal nucleus are seen in $\boldsymbol{e}$ and $\boldsymbol{e}^{\prime}$ but out of view in $\boldsymbol{d}$. Scale bars: $\boldsymbol{b}, \boldsymbol{d}, 20 \mu \mathrm{m} ; \boldsymbol{c}-\boldsymbol{e}^{\prime}, 50 \mu \mathrm{m}$.

axonal projections lay in the REN or CEN suggests that OLe neurons migrate properly without DCC and that our composite map (Fig. 5c) is still valid for morphant cells. Third, these results suggest that DCC is not required for OLe axon guidance.

\section{Discussion}

Our data show that DCC receptor is expressed in OLe neurons at the time that these neurons send their contralateral dendrites toward the midline. Furthermore, its ligands netrin $1 a$ and netrin $1 b$ are expressed at the midline by the neural tube and floor plate cells, respectively. This pattern of expression suggests a role of these guidance molecules in OLe neurons. Indeed, if either the ligand or the receptor is knocked down using antisense MOs, the contralateral dendrites are reduced severely or fail to cross the midline. This failure to cross the midline is not a result of misspecification of OLe neurons because they retain their correct axonal projections. Instead, Netrin/DCC signaling is required for growth of contralateral dendrites across the midline.

Cell transplant experiments show that DCC acts cell autonomously in the OLe neurons. When we transplanted WT cells into DCC-MO1 morphants, we found that $81 \%$ of rhombomeres with isll:gfp cells had dendritic commissures that reached or crossed the midline, very similar to the $82 \%$ observed in WT $>$ WT transplants, showing that normal levels of DCC in the OLe neurons were sufficient to allow their contralateral dendrites to pathfind correctly, even in a DCC-deficient environment. Moreover, when we transplanted cells from DCC-MO1 morphants into wild-type embryos, only $21 \%$ of the rhombomeres with isll:gfp cells had projections that reached or crossed the midline, showing that DCC in the environment is not sufficient for these dendrites to pathfind normally.

The attraction of OLe contralateral dendrites to the midline must happen in a very temporally and spatially regulated manner. Although netrin $1 a$ and netrin $1 b$ are expressed at the midline throughout the migration of REN cells from $\mathrm{r} 4$ to $\mathrm{r} 6$ and CEN cells from $r 6$ to $r 7$, contralateral dendrites are usually only formed after migration, suggesting that the OLe cells are programmed to migrate first to their final positions and only then become sensitive to Netrin 1 from the midline. This implies that Netrin1/DCC signaling is not important for migration of the REN and CEN cell bodies; rather it is only used as their contralateral dendrites grow toward the midline. Furthermore, once OLe contralateral dendrites cross the midline, they must in turn somehow become desensitized to the midline attractant because they travel past the area of netrin 1 expression to reach their final destination. This process could potentially be facilitated by the unc 5 
genes, which are known to convert the Netrin/DCC interaction from attractant to repulsion (Hedgecock et al., 1990; Hong et al., 1999; Keleman and Dickson, 2001). Our collaborators and we have recently cloned all of the zebrafish Unc5 homologues (K. W. Park, A. Suli, C. B. Chien, and D. Y. Li, unpublished observations), and Unc5da is a very good candidate because its expression is detected in the OLe and facial motor neurons at $36 \mathrm{hpf}$ (our unpublished observations). Such a receptor could also play a role in keeping the ipsilateral dendrites and axon of the OLe neurons from being attracted by the midline.

Our experiments have shown that Netrin 1 is important in crossing of the midline by the contralateral OLe dendrites. Because we did not unambiguously visualize single OLe neurons, it was not possible to distinguish between dendritogenesis and dendritic guidance; we cannot rule out the possibility that contralateral dendrites fail to form in our morphants. Time-lapse experiments on single transplanted cells deficient for DCC would be needed to address this question definitively.

It has been shown recently in several systems that ligandreceptor pairs that act to guide axons can guide dendrites as well (Kim and Chiba, 2004). For example, in mice, Sema3A is a chemoattractant for dendrites of pyramidal neurons (Polleux et al., 2000). At the Drosophila midline, Slit/Robo signaling repels dendrites of RP2 neurons (Furrer et al., 2003), whereas Netrin/Frazzled signaling attracts the dendrites of aCC motor neurons. Together with our findings, this latter result shows that a role for Netrin/DCC signaling in dendritic guidance is conserved between invertebrates and vertebrates.

OLe neurons relay bilaterally derived information in many vertebrates studied (Roberts and Meredith, 1989, 1992), but the transmission of contralateral information is accomplished differently in different species. In zebrafish, bilateral information is gathered by having both contralateral and ipsilateral dendrites (Chandrasekhar et al., 1997). In chick (Simon and Lumsden, 1993), a subset of the OLe cell bodies migrates across the midline after generating axons then forms dendrites on the new side to generate a crossed connection. In mice (Fritzsch, 1996), a subset of OLe neurons send axons across the midline to relay contralateral information. It is tempting to speculate that the Ole neurons of different species might use the same guidance molecules but deploy them in different subcellular domains (dendrite, cell body, or axon). It would be interesting to analyze OLe migration in chick, or OLe axon guidance in mouse, after manipulating Netrin/DCC signaling.

The only other guidance molecules known to be involved in the guidance of OLe processes are the Ephrin/Eph family. A report from Cowan et al. (2000) implicates EphB2/EphB3, which are expressed in mouse vestibulo-acoustic axons, in initially attracting these axons toward the midline. EphB2/EphB3 compound homozygotes show a delay of the OLe contralateral axons in reaching the midline at embryonic day 12.5 , as well as some misrouted axons which fail to leave the midline, turning and continuing to grow caudally. The delay and pathfinding mistakes are not seen at later stages (embryonic day 14.5), which suggests that other mechanisms take over for the guidance of the axons. Thus, it could be that OLe neurons use DCC and Netrin 1 to guide their dendrites and EphB/EphrinB to guide their axons across the midline.

Our work has provided the first evidence that the axon guidance molecules DCC and Netrin 1 are required for dendrites in a vertebrate system. This and other studies that implicate axon guidance molecules in dendritic guidance pose the challenge of understanding how guidance molecules can differentially affect axons and dendrites of the same cell.

\section{References}

Bricaud O, Chaar V, Dambly-Chaudiere C, Ghysen A (2001) Early efferent innervation of the zebrafish lateral line. J Comp Neurol 434:253-261.

Chan SS, Zheng H, Su MW, Wilk R, Killeen MT, Hedgecock EM, Culotti JG (1996) UNC-40, a C. elegans homolog of DCC (Deleted in Colorectal Cancer), is required in motile cells responding to UNC-6 netrin cues. Cell 87:187-195.

Chandrasekhar A (2004) Turning heads: development of vertebrate branchiomotor neurons. Dev Dyn 229:143-161.

Chandrasekhar A, Moens CB, Warren Jr JT, Kimmel CB, Kuwada JY (1997) Development of branchiomotor neurons in zebrafish. Development 124:2633-2644.

Cowan CA, Yokoyama N, Bianchi LM, Henkemeyer M, Fritzsch B (2000) EphB2 guides axons at the midline and is necessary for normal vestibular function. Neuron 26:417-430.

Dickson BJ (2002) Molecular mechanisms of axon guidance. Science 298:1959-1964.

Fearon ER, Cho KR, Nigro JM, Kern SE, Simons JW, Ruppert JM, Hamilton SR, Preisinger AC, Thomas G, Kinzler KW, Vogelstein B (1990) Identification of a chromosome $18 \mathrm{q}$ gene that is altered in colorectal cancers. Science 247:49-56.

Fricke C, Chien CB (2005) Cloning of full-length zebrafish dcc and expression analysis during embryonic and early larval development. Dev Dyn 234:732-739.

Fritzsch B (1996) Development of the labyrinthine efferent system. Ann NY Acad Sci 781:21-33.

Furrer MP, Kim S, Wolf B, Chiba A (2003) Robo and Frazzled/DCC mediate dendritic guidance at the CNS midline. Nat Neurosci 6:223-230.

Godenschwege TA, Simpson JH, Shan X, Bashaw GJ, Goodman CS, Murphey RK (2002) Ectopic expression in the giant fiber system of Drosophila reveals distinct roles for roundabout (Robo), Robo2, and Robo3 in dendritic guidance and synaptic connectivity. J Neurosci 22:3117-3129.

Hedgecock EM, Culotti JG, Hall DH (1990) The unc-5, unc-6, and unc-40 genes guide circumferential migrations of pioneer axons and mesodermal cells on the epidermis in C. elegans. Neuron 4:61-85.

Hedrick L, Cho KR, Fearon ER, Wu TC, Kinzler KW, Vogelstein B (1994) The DCC gene product in cellular differentiation and colorectal tumorigenesis. Genes Dev 8:1174-1183.

Higashijima S, Hotta Y, Okamoto H (2000) Visualization of cranial motor neurons in live transgenic zebrafish expressing green fluorescent protein under the control of the islet-1 promoter/enhancer. J Neurosci 20:206-218.

Hjorth JT, Gad J, Cooper H, Key B (2001) A zebrafish homologue of deleted in colorectal cancer ( $\mathrm{zdcc}$ ) is expressed in the first neuronal clusters of the developing brain. Mech Dev 109:105-109.

Hong K, Hinck L, Nishiyama M, Poo MM, Tessier-Lavigne M, Stein E (1999) A ligand-gated association between cytoplasmic domains of UNC5 and DCC family receptors converts netrin-induced growth cone attraction to repulsion. Cell 97:927-941.

Kane DA, Kishimoto Y (2002) Cell labelling and transplantation techniques. In: Zebrafish: a practical approach (Nüsslein-Volhard C, Dahm R, eds), pp 95-119. Oxford: Oxford UP.

Keino-Masu K, Masu M, Hinck L, Leonardo ED, Chan SS, Culotti JG, TessierLavigne M (1996) Deleted in Colorectal Cancer (DCC) encodes a netrin receptor. Cell 87:175-185.

Keleman K, Dickson BJ (2001) Short- and long-range repulsion by the Drosophila Unc5 netrin receptor. Neuron 32:605-617.

Kim S, Chiba A (2004) Dendritic guidance. Trends Neurosci 27:194-202.

Kimmel CB, Ballard WW, Kimmel SR, Ullmann B, Schilling TF (1995) Stages of embryonic development of the zebrafish. Dev Dyn 203:253-310.

Lauderdale JD, Davis NM, Kuwada JY (1997) Axon tracts correlate with netrin-1a expression in the zebrafish embryo. Mol Cell Neurosci 9:293-313.

Metcalfe WK, Kimmel CB, Schabtach E (1985) Anatomy of the posterior lateral line system in young larvae of the zebrafish. J Comp Neurol 233:377-389.

Moens CB, Fritz A (1999) Techniques in neural development. In: The zebrafish: biology (Methods in cell biology) (Detrich III WH, Westerfield M, Zon LI, eds), pp 264-267. New York: Academic.

Park KW, Urness LD, Senchuk MM, Colvin CJ, Wythe JD, Chien CB, Li DY 
(2005) Identification of new netrin family members in zebrafish: developmental expression of netrin2 and netrin4. Dev Dyn 234:726-731.

Polleux F, Morrow T, Ghosh A (2000) Semaphorin 3A is a chemoattractant for cortical apical dendrites. Nature 404:567-573.

Roberts BL, Meredith GE (1989) The efferent system. In: The mechanosensory lateral line: neurobiology and evolution (Coombs S, Görner P, Münz H, eds), pp 445-459. New York: Springer.

Roberts BL, Meredith GE (1992) The efferent Innervation of the ear: variation on an enigma. In: The evolutionary biology of hearing (Webster DB, Fay RR, Popper AN, eds), pp 185-210. New York: Springer.

Sapede D, Rossel M, Dambly-Chaudiere C, Ghysen A (2005) Role of SDF1 chemokine in the development of lateral line efferent and facial motor neurons. Proc Natl Acad Sci USA 102:1714-1718.
Simon H, Lumsden A (1993) Rhombomere-specific origin of the contralateral vestibulo-acoustic efferent neurons and their migration across the embryonic midline. Neuron 11:209-220.

Strahle U, Fischer N, Blader P (1997) Expression and regulation of a netrin homologue in the zebrafish embryo. Mech Dev 62:147-160.

Tomasiewicz HG, Wood JG (1999) Characterization of microtubuleassociated proteins in teleosts. Cell Motil Cytoskeleton 44:155-167.

Xiao T, Roeser T, Staub W, Baier H (2005) A GFP-based genetic screen reveals mutations that disrupt the architecture of the zebrafish retinotectal projection. Development 132:2955-2967.

Zottoli SJ, Van Horne C (1983) Posterior lateral line afferent and efferent pathways within the central nervous system of the goldfish with special reference to the Mauthner cell. J Comp Neurol 219:100-111. 